$(S)$ - and $(R)$-Fmoc- $\alpha-M e C y s(\operatorname{Trt})-\mathrm{OH}$

Supporting Information

\title{
List of the contents of the supporting information
}

$\begin{array}{ll}\text { Material and Methods } & \text { S-2 }\end{array}$

(1S)-(-)-2,10- $N$-(2-Phenylthiazoline-4-carbonyl)camphorsultam (20) S-3

(1S)-(+)-2,10-N-((4R)-Methyl-2-phenylthiazoline-4-carbonyl)camphorsultam (21): $\quad$ S-3

$(R)-S$-Trityl- $\alpha$-methylcysteine (22): $\quad$ S-4

(R)-N-Fmoc-S-trityl- $\alpha$-methylcysteine (23): $\quad$ S-5

$\begin{array}{ll}\text { References } & \text { S-5 }\end{array}$ 
$(S)$ - and $(R)$-Fmoc- $\alpha-M e C y s(\operatorname{Trt})-\mathrm{OH}$

Supporting Information

\title{
An Efficient Asymmetric Synthesis of (S)- and (R)- $N$-Fmoc- $S$-trityl- $\alpha$ - methylcysteine Using Camphorsultam as a Chiral Auxiliary
}

\author{
Satendra Singh, ${ }^{*}$ Samala J. Rao, and Michael W. Pennington \\ Bachem Bioscience Inc., 3700 Horizon Drive, King of Prussia, PA 19406
}

\section{Supporting Information}

Materials and Methods: All the reagents and solvents were used without further purification unless otherwise stated. Methyl iodide was freshly distilled. THF was dried over LAH and freshly distilled before use. HMPA was dried over $4 \AA$ molecular sieves.

HPLC was performed on a $\mathrm{C}_{18}$, reversed phase column (Vydac, 250 x $4.6 \mathrm{mM}, 5 \mu$ ). A linear gradient from $5 \%$ buffer B to $95 \%$ buffer B in 45 min was used. Buffer A consisted of $0.1 \%$ TFA in water and buffer B was MeCN containing $0.1 \%$ TFA. Flow rate was $1.5 \mathrm{~mL} / \mathrm{min}$.

The mass spectra of crude and purified samples were obtained using in-house MALDIMS (Kratos Analytical, A shimadzu Group Company, New York)) and ESI-MS (HT laboratories, San Diego, CA). For MALDI-MS, $2 \mu \mathrm{L}$ of the peptide sample was spotted on a 15well plate and ionized with a laser beam from a nitrogen laser (337 nm) on a MicroMass MALDI-TOF instrument. The sample was prepared by mixing $2 \mu \mathrm{L}$ of $1 \mathrm{mg} / \mathrm{mL}$ solution of compound with $18 \mu \mathrm{L}$ of a saturated solution of $\alpha$-cyano-4-hydroxycinnamic acid matrix. ESI-

MS spectra were collected from samples dissolved in methanol as a solvent. The ${ }^{1} \mathrm{H}$ and ${ }^{13} \mathrm{C}$ NMR spectra were obtained using a $600 \mathrm{MHz}$ Varian Unity600 NMR spectrometer with $\mathrm{CDCl}_{3}$ 
$(S)$ - and $(R)$-Fmoc- $\alpha-M e C y s(T r t)-O H$

Supporting Information

or $\mathrm{d}_{6}$-DMSO as solvent (Emory University, Atlanta, GA). Micro Analysis Inc. (Wilmington, DE) performed elemental analysis.

Diastereoisomeric excess (asymmetric induction) during the alkylation step was measured using HPLC and NMR spectrometer. Optical rotation measurements on purified samples were obtained from Bachem AG, Bubendorf, Switzerland.

(1S)-(-)-2,10-N-(2-Phenylthiazoline-4-carbonyl)camphorsultam (20): A 2M solution of $\mathrm{Me}_{3} \mathrm{Al}(14 \mathrm{~mL}, 27.9 \mathrm{mmol})$ was added dropwise to a suspension of (1S)-camphorsultam, enantiomer of $12(5.0 \mathrm{~g}, 23.25 \mathrm{mmol})$ in toluene $(50 \mathrm{~mL}) .{ }^{1}$ The mixture was heated to reflux. After 5 min of refluxing, the clear solution was allowed to cool to ambient temperature over the period of $30 \mathrm{~min}$ and continued to stir for additional $30 \mathrm{~min}$. A solution of thiazoline 11 (6.55 g, $27.9 \mathrm{mmol})$ in toluene $(30 \mathrm{~mL})$ was added. The reaction mixture turned bright yellow immediately upon addition. It was stirred at $55 \pm 5{ }^{\circ} \mathrm{C}$ for $24 \mathrm{~h}$. The mixture was cooled in an ice bath and $\mathrm{MeOH}(20 \mathrm{~mL})$ was added dropwise. After stirring for additional $30 \mathrm{~min}$, water (20 $\mathrm{mL}$ ) was added dropwise. After stirring for $60 \mathrm{~min}$, the mixture was filtered through Celite and washed with EtOAc $(300 \mathrm{~mL})$. The filtrate and the washings were combined, dried over $\mathrm{MgSO}_{4}$, and concentrated in vacuo to afford a syrup (14 g). Purification of the crude product by silica gel chromatography, EtOAc/Hexane (5:95 to 20:80) afforded 6.8 g (72\%) of white solid. Mp 168$169{ }^{\circ} \mathrm{C}$. RP-HPLC: $t_{\mathrm{R}}=28.1 \mathrm{~min} .[\alpha]_{\mathrm{D}}{ }^{24}=-71.37\left(\mathrm{c} 0.413, \mathrm{CHCl}_{3}\right)$. ESI-MS analysis gave a molecular ion peak at $405\left(\mathrm{C}_{20} \mathrm{H}_{25} \mathrm{~N}_{2} \mathrm{O}_{3} \mathrm{~S}_{2}\right)(\mathrm{M}+\mathrm{H})^{+} .{ }^{1} \mathrm{H} \mathrm{NMR}\left(\mathrm{CDCl}_{3}\right): \delta 7.88(2 \mathrm{H}, \mathrm{d}, J=7.5$ Hz), $7.42(1 \mathrm{H}, \mathrm{m}), 7.40(2 \mathrm{H}, \mathrm{m}), 5.87(1 \mathrm{H}, \mathrm{t}, J=6.9 \mathrm{~Hz}), 4.02(1 \mathrm{H}, \mathrm{m}), 3.72(1 \mathrm{H}, \mathrm{d}, J=7.2 \mathrm{~Hz})$, 3.62-3.42 (3H, m), 2.18-2.06 (2H, m), 1.98-1.85 (3H, m), 1.50-1.42 (1H, m), 1.40-1.32 (1H, m), $1.16(3 \mathrm{H}, \mathrm{s}), 0.98(3 \mathrm{H}, \mathrm{s}) .{ }^{13} \mathrm{C} \mathrm{NMR}\left(\mathrm{DMSO}-d_{6}\right): \delta 170.3,168.5,132.1,132.0,128.9,128.2$, 
$(S)$ - and $(R)$-Fmoc- $\alpha-M e C y s(T r t)-O H$

Supporting Information

64.2, 52.0, 48.7, 47.4, 44.5, 37.6, 31.8, 25.8, 20.1, 19.4. Anal. Calcd for $\mathrm{C}_{20} \mathrm{H}_{24} \mathrm{~N}_{2} \mathrm{O}_{3} \mathrm{~S}_{2}$ : C, 59.40;

H, 5.94; N, 6.90. Found: C, 59.16, H, 5.74; N, 6.67.

(1S)-(+)-2,10-N-((4R)-Methyl-2-phenylthiazoline-4-carbonyl)camphorsultam (21): A

$1.6 \mathrm{M} n$-BuLi solution in hexane $(0.55 \mathrm{~mL}, 8.72 \mathrm{mmol})$ was added over $30 \mathrm{~min}$ at $-78{ }^{\circ} \mathrm{C}$ to a stirred solution of 20 ( $3.2 \mathrm{~g}, 7.92 \mathrm{mmol})$ in dry THF (60 mL). After stirring for $90 \mathrm{~min}$ at the same temperature, a solution of MeI (3.38 mL, $23.76 \mathrm{mmol})$, HMPA (4.2 mL, $23.76 \mathrm{mmol})$ in THF (5 mL) was added over $30 \mathrm{~min}$. After stirring for additional $90 \mathrm{~min}$ at $-78{ }^{\circ} \mathrm{C}$, the reaction mixture was allowed to warm to ambient temperature over $2 \mathrm{~h}$. After cooling in ice bath the reaction was quenched with $3.7 \mathrm{M}$ aqueous $\mathrm{NH}_{4} \mathrm{Cl}(12 \mathrm{~mL})$. The mixture was taken in $250 \mathrm{~mL}$ of EtOAc and successively washed with $10 \%$ aqueous citric acid $(3 \times 60 \mathrm{~mL})$, saturated aqueous $\mathrm{NaHCO}_{3}(3 \times 60 \mathrm{~mL}), \mathrm{H}_{2} \mathrm{O}(2 \times 60 \mathrm{~mL})$, and brine $(2 \times 60 \mathrm{~mL})$. The organic layer was dried over $\mathrm{MgSO}_{4}$ and concentrated in vacuo to afford $3.5 \mathrm{~g}$ red oil. The crude mixture was purified by silica gel chromatography, EtOAc/hexane (5:95 to 20:80) to furnish $1.62 \mathrm{~g}$ (49\%) white solid. Mp 138-140 ${ }^{\circ} \mathrm{C}$. RP-HPLC: $t_{\mathrm{R}}=29.93 \mathrm{~min} .[\alpha]_{\mathrm{D}}{ }^{24}=-86.67$ (c $\left.0.15, \mathrm{MeOH}\right)$. ESI-MS analysis gave a molecular ion peak at $419\left(\mathrm{C}_{21} \mathrm{H}_{27} \mathrm{~N}_{2} \mathrm{O}_{3} \mathrm{~S}_{2}\right)(\mathrm{M}+\mathrm{H})^{+} .{ }^{1} \mathrm{H} \mathrm{NMR}\left(\mathrm{CDCl}_{3}\right): \delta 7.92(2 \mathrm{H}, \mathrm{d}, J=$ $7.5 \mathrm{~Hz}), 7.43(1 \mathrm{H}, \mathrm{m}), 7.40(2 \mathrm{H}, \mathrm{m}), 4.17-4.10(1 \mathrm{H}, \mathrm{m}), 3.58-3.26(3 \mathrm{H}, \mathrm{m}), 2.92(1 \mathrm{H}, \mathrm{m}), 2.50$ $(3 \mathrm{H}, \mathrm{s}), 1.94-1.80(5 \mathrm{H}, \mathrm{m}), 1.46-1.40(1 \mathrm{H}, \mathrm{m}), 1.25-1.21(1 \mathrm{H}, \mathrm{m}), 1.14(3 \mathrm{H}, \mathrm{s}), 0.90(3 \mathrm{H}, \mathrm{s}) .{ }^{13} \mathrm{C}$ NMR (DMSO- $\left.d_{6}\right): \delta 172.8,126.2,132.5,131.6,128.5,128.4,67.7,49.5,48.4,47.2,43.7,34.0$, 31.5, 27.1, 26.5, 20.0, 19.6. Anal. Calcd for $\mathrm{C}_{21} \mathrm{H}_{26} \mathrm{~N}_{2} \mathrm{O}_{3} \mathrm{~S}_{2}: \mathrm{C}, 60.28 ; \mathrm{H}, 6.22 ; \mathrm{N}, 6.69$. Found: C, 60.10, H, 5.98; N, 6.42 .

(R)-S-Trityl- $\alpha$-methylcysteine (22): Sultam $21(1.0 \mathrm{~g}, 2.4 \mathrm{mmol})$ was refluxed in $6 \mathrm{~N}$ $\mathrm{HCl}(18 \mathrm{~mL})$ for $8 \mathrm{~h}$. The mixture was washed with EtOAc $(3 \times 10 \mathrm{~mL})$ and evaporated under reduced pressure. The syrup was taken in $\mathrm{EtOH}$ and treated with MTBE to yield a white solid 
$(S)$ - and $(R)$-Fmoc- $\alpha-M e C y s(T r t)-O H$

Supporting Information

$(1.1 \mathrm{~g})$. Without further purification, the solid was treated with trityl alcohol $(0.75 \mathrm{~g}, 2.9 \mathrm{mmol})$ and $\mathrm{BF}_{3} . \mathrm{OEt}_{2}(0.42 \mathrm{~mL})$ in glacial acetic acid $(8 \mathrm{~mL})$ at $80+5{ }^{\circ} \mathrm{C}$ for $30 \mathrm{~min}$ according to reported procedure. ${ }^{2}$ After work up and purification by silica gel chromatography, $0.45 \mathrm{~g}$ of a white solid (49\% over two steps) was obtained. Mp 179-180 ${ }^{\circ} \mathrm{C}$. RP-HPLC: $t_{\mathrm{R}}=20.48 \min$. $[\alpha]_{\mathrm{D}}{ }^{24}=+28.5(\mathrm{c} 0.15, \mathrm{MeOH})$. ESI-MS analysis gave a molecular ion peak at 378 $\left(\mathrm{C}_{23} \mathrm{H}_{24} \mathrm{NO}_{2} \mathrm{~S}\right)(\mathrm{M}+\mathrm{H})^{+} .{ }^{1} \mathrm{H}$ NMR $\left(\mathrm{DMSO}-d_{6}\right): \delta 7.39-7.14(15 \mathrm{H}, \mathrm{m}), 2.41(1 \mathrm{H}, \mathrm{d}, J=6.0 \mathrm{~Hz})$ $2.33(1 \mathrm{H}, \mathrm{d}, J=6.0 \mathrm{~Hz}), 1.14(3 \mathrm{H}, \mathrm{s}) .{ }^{13} \mathrm{C}$ NMR $\left(\mathrm{DMSO}-d_{6}\right): \delta 170.3,144.2,129.0,128.0,126.7$, 65.7, 58.8, 22.1. Anal. Calcd for $\mathrm{C}_{23} \mathrm{H}_{23} \mathrm{NO}_{2} \mathrm{~S}$ : C, 73.21; H, 6.10; N, 3.71. Found: C, 72.98, H, $6.01 ; \mathrm{N}, 3.43$.

(R)-N-Fmoc-S-trityl- $\alpha$-methylcysteine (23): Compound 22 (0.075 g, $0.2 \mathrm{mmol})$ was treated with Fmoc-OSu $(0.080 \mathrm{~g}, 0.22 \mathrm{mmol})$ in the presence of $\mathrm{Na}_{2} \mathrm{CO}_{3}(0.045 \mathrm{~g}, 0.4 \mathrm{mmol})$ in water/dioxane mixture $(10 \mathrm{~mL})$ overnight according to reported procedure. ${ }^{3}$ After work up, 0.12 $\mathrm{g}(96 \%)$ of a white soild was obtained. Mp 182-183 ${ }^{\circ} \mathrm{C}$. RP-HPLC: $t_{\mathrm{R}}=35.4 \mathrm{~min} .[\alpha]_{\mathrm{D}}{ }^{24}=+31.9$ (c $0.25, \mathrm{MeOH})$. ESI-MS analysis gave a molecular ion peak at $600\left(\mathrm{C}_{38} \mathrm{H}_{34} \mathrm{NO}_{4} \mathrm{~S}\right)(\mathrm{M}+\mathrm{H})^{+} .{ }^{1} \mathrm{H}$ NMR (DMSO-d $\left.d_{6}\right): \delta$ 7.90-7.85 $(2 \mathrm{H}, \mathrm{m}), 7.72-7.67(2 \mathrm{H}, \mathrm{m}), 7.40-7.35(2 \mathrm{H}, \mathrm{m}), 7.28(15 \mathrm{H}, \mathrm{s})$, 7.22-7.15 (2H, m), 4.30-4.15 (3H, br, m), 2.88-2.68 (2H, m), 1.47 (3H, s). ${ }^{13} \mathrm{C}$ NMR (DMSO- $\left.d_{6}\right)$ : $\delta 174.8,154.1,144.6,143.9,140.6,129.1,127.8,127.6,127.1,127.0,126.5,125.3,120.1,65.3$, 65.0, 57.9, 46.7, 23.3. Anal. Calcd for $\mathrm{C}_{38} \mathrm{H}_{33} \mathrm{NO}_{4} \mathrm{~S}$ : C, 76.12; H, 5.50; $\mathrm{N}, 2.33$. Found: C, 75.98, H, 5.45; N, 2.17.

\section{References}

1. Oppolzer, W.; Moretti, R.; Zhou, C. Helv. Chim. Acta 1994, 77, 2363-2380. 
$(S)$ - and $(R)$-Fmoc- $\alpha-M e C y s(\operatorname{Trt})-\mathrm{OH}$

Supporting Information

2. Bodanszky, M.; Bodanszky, A. In The Practice of Peptide Synthesis, $2^{\text {nd }}$ ed.; SpringerVerlag Berlin Heidelberg, Germany, 1994; pp. 68-69.

3. Atherton, E.; Sheppard, R.C. In Solid Phase Peptide Synthesis, IRL Press, Oxford, England, 1989, pp. 61. 